\title{
Choix rationnel, frustration et protestation sociale
}

Le cas des diplômés chômeurs au Maroc

Rational choice, deprivation and social protest : the case of unemployed graduates in Morocco

\section{Abderrahmane Rachik}

\section{OpenEdition \\ Journals}

Édition électronique

URL : http://journals.openedition.org/ress/3596

DOI : $10.4000 /$ ress.3596

ISBN : 1663-4446

ISSN : $1663-4446$

Éditeur

Librairie Droz

\section{Édition imprimée}

Date de publication : 30 novembre 2016

Pagination : 195-218

ISSN : 0048-8046

Référence électronique

Abderrahmane Rachik, "Choix rationnel, frustration et protestation sociale », Revue européenne des sciences sociales [En ligne], 54-2 | 2016, mis en ligne le 30 novembre 2019, consulté le 02 janvier 2020. URL : http://journals.openedition.org/ress/3596 ; DOI : 10.4000/ress.3596 


\title{
CHOIX RATIONNEL, FRUSTRATION ET PROTESTATION SOCIALE
}

\author{
LE CAS DES DIPLÔMÉS CHÔMEURS AU MAROC
}

\author{
ABDERRAHMANE RACHIK \\ Université Mohammed VI Polytechnique, Rabat \\ rachik1956@gmail.com
}

\begin{abstract}
Résumé. Le mouvement social des diplômés chômeurs au Maroc est né dans un contexte politique autoritaire. II s'est imposé comme interlocuteur face aux pouvoirs publics grâce à sa mobilisation récurrente et à sa stratégie de perturbation de l'ordre établi dans la capitale du pays. Le recours au paradigme du choix rationnel permet d'expliquer la réussite et la mobilisation permanente du mouvement dans un système politique en changement. Quant à la théorie de la frustration relative et à celle de la mobilisation des ressources, elles offrent pour leur part un autre angle d'attaque pour suivre pertinemment les premiers pas du mécontentement ainsi que le processus d'évolution des formes de protestation sociale.
\end{abstract}

Mots-clés: choix rationnel, diplômés chômeurs, frustration, Maroc, mouvement social, opportunités politiques

\begin{abstract}
The social movement of unemployed graduates in Morocco was born in an authoritarian political context. It has become an interlocutor to the public authorities thanks to its recurrent mobilization and its strategy of disturbing established order in the country's capital. The use of a rational choice paradigm can explain the success and permanent mobilization of the movement in a changing political system. While the theories of relative deprivation and resource mobilization offer another angle to efficiently keep track of the discontent and the evolutionary process characteristic of forms of social mobilization.
\end{abstract}

Keywords: deprivation, Morocco, political opportunities, rational choice, social movement, unemployed graduates. 
Le suivi du mouvement des diplômés universitaires en chômage permet d’analyser l'évolution de la protestation sociale au Maroc (Rachik, 20I4). Depuis sa création en I990, ce mouvement social s’est développé dans un contexte politique autoritaire où les protestations dans l'espace public n'ont presque jamais été autorisées, malgré l'existence d’un Code des libertés publiques libéral qui reconnaît, depuis 1958, la grève et la manifestation. Puis, le mouvement a par la suite évolué dans un système politique qui s'est ouvert progressivement en passant par d'autres expériences politiques : gouvernement dit « d’alternance ${ }^{1} »$, à partir de 1998, puis gouvernement islamiste, à partir de 20I2. Les représentants des groupes des diplômés chômeurs (DC) se sont imposés comme l'intermédiaire incontournable face aux pouvoirs publics.

Les jeunes DC étaient les premiers à exprimer régulièrement et massivement leurs protestations sociales à travers des sit-in, des marches et des grèves de la faim dans l'espace public, notamment devant les sièges des ministères de l'Éducation nationale, de l'Intérieur, de l'Emploi, devant le Parlement, des préfectures, ou tout simplement dans la rue.

La question que nous nous posons est liée à la permanence et à la récurrence de l'action collective du mouvement des DC dans l'espace public. Notre préoccupation initiale n'était pas de tester ou de valider une quelconque hypothèse théorique. C'est notre démarche empirique qui nous a permis de recourir aux différents paradigmes et théories relatifs au choix rationnel, à la frustration relative et à la mobilisation des ressources qui sont susceptibles de rendre compte du comportement protestataire des DC.

En effet, le paradigme du choix rationnel permet d'expliquer la réussite et surtout, la récurrence de la mobilisation sociale des DC. L'organisation de leur action collective parvient à éliminer le risque de «ticket gratuit» ou de «free rider », c'est-à-dire qu'elle interdit au DC qui reste à l'écart de la mobilisation collective de bénéficier des ressources collectivement revendiquées (l’emploi). Le recours au paradigme du choix rationnel pour analyser le cas étudié est une

I Après des décennies passées à l'opposition, la gauche accède au gouvernement, mené par le parti de l'Union socialiste des forces populaires (USFP). 
phase pertinente dans l'approche de l'action collective. Mais ce paradigme doit être intégré dans un contexte politique, social et économique qui constitue le cadre structurant de l'activité protestataire. Les opportunités politiques à l'échelle locale et nationale, voire internationale (Printemps arabe en 20I I), peuvent donc entraver ou favoriser la formation et le développement de l'action collective.

Au niveau macro et micro, tous les ingrédients sont réunis pour assurer la construction d'une action collective :

- Le mécontentement des DC accentué par la peur d’un éventuel déclassement social;

- les opportunités politiques favorables à la mobilisation sociale: ouverture du système politique et engagement d'un dialogue officiel avec les protestataires ;

- des revendications clairement formulées relatives à la quête d'emploi ;

- la capacité de mobilisation collective: le mouvement bénéficie du soutien de plusieurs organisations politiques, syndicales et associatives;

- l'accès aux mass-média classiques et aux nouvelles technologies.

En effet, au début de sa constitution à Casablanca, l'Association nationale des DC au Maroc (ANDCM) a attiré des centaines de jeunes qui ont déposé leurs dossiers de candidature à l'emploi. Mais quand il s'est agi de s'organiser, de mener des réunions et d'établir un agenda d'actions protestataires, la participation était trop faible. Seuls certains militants chevronnés, dont l'intérêt ne se réduisait pas uniquement à l'obtention d'un emploi, ont assisté aux réunions au siège du syndicat de la Confédération démocratique du travail (CDT). Le contexte politique autoritaire n’a guère favorisé le passage du mécontentement individuel à une action collective. Et les militants du mouvement avaient donc du mal à mobiliser les jeunes diplômés en situation de chômage.

Selon notre hypothèse, si le paradigme du choix rationnel permet d'expliquer la mobilisation sociale récurrente des DC, la théorie de la frustration, fournit un autre angle d’attaque. Nous recourons à cette théorie, développée durant les années 1960 principalement par James Davies (1997) et Ted Robert Gurr (20I0), parce qu'elle nous permet d'éclairer l'émergence du 
mécontentement chez le DC. Le sentiment du mécontentement est la première condition pour la construction d'une action collective. Mais le mécontentement à lui seul ne suffit pas pour aboutir à une mobilisation collective.

À noter que le mécontentement n'est pas corrélé à des facteurs économiques déterminants (chômage, pauvreté, crise économique, etc.), mais qu'il est pensé comme un décalage entre les attentes socialement construites et la réalité de la situation présente. En effet, chez le DC, la frustration est doublement ressentie: la non-satisfaction espérée d'une promotion sociale après l'obtention d'un diplôme universitaire se voit renforcée par un sentiment d'injustice (frustration relative) par rapport aux autres DC qui ont déjà bénéficié d'un emploi dans la fonction publique. Nous émettons donc l'hypothèse selon laquelle la frustration relative des DC explique le processus de radicalisation de leur mouvement.

Sans vouloir adopter un schéma linéaire ou un classement hiérarchique des comportements collectifs, nous remarquons que l'action protestataire urbaine passe de l'émeute violente, au cours des années 1980, dans un contexte politique autoritaire, aux sit-in pacifiques à partir du début des années 1990. Jusqu’ici, les rares sit-in et manifestations organisés revêtaient un caractère plutôt politique que social. Les revendications à caractère social étaient limitées aux seuls lieux de travail et espaces scolaires et universitaires. La stratégie consistant à occuper la rue n’est devenue un enjeu politique de taille quaprès 1994, année qui correspond à un début d'ouverture du système politique. À noter que les changements politiques se sont essentiellement caractérisés par l'adoption d'une nouvelle Constitution en 1992. Celle-ci a mis en exergue l'attachement du Maroc au respect des droits de l'homme tels qu'ils sont reconnus universellement. Depuis, les demandes d’autorisation de manifester se sont multipliées. La manifestation, comme action collective organisée et autorisée, fait ainsi progressivement son entrée dans le répertoire de l'action collective nationale.

Pour instaurer progressivement la tradition de l'action manifestante, la mobilisation sociale des syndicats et des partis politiques de l'opposition, au début des années 1990, s'appuyait d’abord sur l'émotion collective en appelant à se solidariser avec les peuples en guerre, les Palestiniens, les Libanais, les Irakiens, etc. En effet, dans un contexte politique autoritaire, les revendica- 
tions autour d'intérêts catégoriels ou d'enjeux politiques internes n'étaient pas susceptibles de mobiliser les masses urbaines.

La période allant de 1990 à 1996 a été la phase préparatoire de l’action manifestante. Cette étape a permis aux protestataires de mettre à l'épreuve l'attitude du pouvoir à travers la réaction des forces chargées de maintenir l'ordre, et d'évaluer ainsi non seulement le coût de l'engagement de l'individu mais également celui des organisations politiques et syndicales dans la conquête de l'espace public. À partir de la fin de 1995, nous notons le passage de l'action collective qui se déroule à l'intérieur des murs (lycée, faculté, usine, bâtiment administratif, sièges des partis et syndicats, etc.), vers la conquête de l'espace public. Même si quelques tentatives d'occupation de l'espace public pouvaient être observées avant cette date, elles ne constituaient encore qu'un phénomène isolé.

C'est dans ce contexte que se construit le mouvement des DC qui allait devenir l'initiateur de la stratégie d'occupation de la rue. Mais, cette fois-ci, son action collective récurrente est menée non pas pour exprimer des opinions ou des émotions, mais pour mettre en relief des revendications exclusivement sociales, à savoir l'obtention d'un emploi.

Trois points fondamentaux ont donc favorisé la massification du mécontentement collectif chez les DC :

- Le processus d'ouverture du système politique qui tolère plus ou moins l'occupation de l'espace public ;

- le soutien actif des organisations politiques, syndicales et associatives, notamment celles des droits de l'homme, sans oublier les mass-média;

- la frustration relative provoquée par le déclenchement d’une politique publique donnant naissance à de nouvelles attentes relatives à l'emploi dans la fonction publique.

À cet égard, le succès symbolique que constituent les différentes réceptions officielles du mouvement, a pour conséquence d’alimenter l'espoir d'une éventuelle embauche dans la fonction publique. En effet, les groupes de DC étaient officiellement reçus par les différents gouverneurs et ministres, par le Premier ministre et plus particulièrement par le roi Hassan II. 
Comme l’a constaté Tocqueville, dans La Démocratie en Amérique, «si certains de mes semblables bénéficient d'un privilège, j'éprouverai un sentiment d'injustice; en revanche quand l'inégalité est la loi commune dans la société ou le groupe où je vis, les inégalités même extrêmes sont supportables » (cité par Cherkaoui, 2005, p. 470). De fait, les premières affectations dans la fonction publique ont augmenté le degré des attentes chez les DC, ainsi que le nombre des protestataires. La frustration relative a renforcé par conséquent le sentiment d’injustice.

\section{DES GROUPES INCLUSIFS}

Toutes les actions collectives des DC ne se déroulent pas dans le cadre de l'Association nationale des diplômés chômeurs qui n'est pas juridiquement reconnue. Après la création de celle-ci, en I99I, de petits groupes (majemoua) se sont multipliés pour tenter de défendre leurs revendications d'une manière autonome par rapport à l'association.

Pour faire partie d'un groupe, le DC doit participer à une série d'actions de protestation. Le groupe se forme par le biais d'une liste close où figurent les noms des participants au sit-in. Ainsi, les nouveaux DC qui n'ont pas pu s'inscrire sur la liste doivent-ils former un nouveau groupe qui assurera à son tour sa propre organisation et ses actions collectives. On se retrouve donc face à plusieurs groupes qui se constituent par affinité, soit en fonction de leurs spécialités académiques, tels les ingénieurs agronomes, soit en fonction de leurs statuts académiques, tels les docteurs, les DESA etc. Ces groupes sont en outre traversés par des courants politiques qui sont, en partie, à la base de leur création. Les différents courants dominant le syndicat étudiant, l’Union nationale des étudiants marocains, ont ainsi investi le champ de la protestation. Dans un même groupe coexistent aussi bien les islamistes radicaux (Al-adle wa al-ihsane) que les militants d'extrême gauche (les Basistes), les islamistes dits modérés (Parti al-Adala wa tanmiya) que des individus sans coloration partisane. 
Il existe également d'autres groupes spécifiques, tels les diplômés non-voyants en chômage ou encore le groupe des diplômés chômeurs (titulaire d'une Licence) qui ont été affectés par le ministère de l'Éducation nationale à des services civils, mais qui, faute de crédits budgétaires, se sont retrouvés sans emploi.

Il s'agit donc d'un groupement disposant d'une organisation interne structurée, mené par des représentants, élus par une assemblée générale, qui s’imposent comme interlocuteurs face aux autorités publiques. Il n'en demeure pas moins que ces groupes sont de nature fugace: une fois embauchés, la plupart de leurs membres disparaissent du champ de la protestation.

Le nombre d'individus composant le groupe est restreint. Il peut se situer entre une vingtaine, comme c'est le cas des chômeurs non-voyants, et une centaine de personnes. En 2008, il existait 25 groupes de DC qui représentaient un ensemble de 3450 personnes $^{2}$, soit une moyenne de i38 personnes par groupe 3 .

En effet, dans le but d'assurer une efficacité de gestion et de mobilisation, la taille des groupes est réduite à une petite dimension. Cependant, il faut être nombreux pour se mettre à l'abri d'éventuelles mesures de représailles, et surtout pour assurer une mobilisation massive et une pression sur les pouvoirs publics. Pour les autorités locales, un petit groupe est relativement facile à contrôler, à disperser ou à réprimer. Il faut aussi signaler que plus la mobilisation est importante, plus le bien revendiqué a davantage de chance d'être obtenu.

C'est dans ce sens qu'un autre niveau d’organisation est créé par les différents groupes pour constituer une fédération informelle. En 2004, l'Union des cadres supérieurs en chômage est créée qui regroupe 560 membres. Elle se compose de trois groupes: des docteurs du troisième cycle, des titulaires

2 Selon la base de données des chômeurs titulaires de troisième cycle (Royaume du Maroc, services du Premier ministre, Rabat, 2007 - Document inédit). II s'agit en fait d'une liste nominative sous forme de tabulation que nous avons convertie en une base de données. Celle-ci regroupe plusieurs listes et recense 3280 DC.

3 À noter que seul un groupe, Al-istihkake, est composé de plus de 1200 personnes. On peut se demander pourquoi lors des sit-in, ce groupe ne mobilise qu'une centaine de personnes! Les concurrents affirment que la liste du groupe est exagérément gonflée pour profiter au mieux des éventuels postes dans la fonction publique. En outre, plusieurs membres inscrits ne font pas actes de présence parce qu'ils exercent un métier dans le secteur privé. 
de diplômes d'études approfondies (DEA) ou de diplômes des études supérieures approfondies (DESA). Elle a organisé une série de sit-in devant le siège du Parlement. Et pour donner davantage de médiatisation à leur action collective, environ 400 personnes rassemblées se sont enchaînées en scandant des slogans relatifs à leur «droit constitutionnel au travail.»

Certes, les protestataires ont des objectifs convergents, à savoir l'obtention d'un emploi dans la fonction publique par le truchement de l'occupation de l'espace public. Mais, objectivement, le bien revendiqué doit être un enjeu de rivalité entre les différents groupes protestataires, l'offre d'emploi dans la fonction publique n'étant pas extensible. Tout en sachant que le nombre d'emplois disponibles est limité, les petits groupes, vu leur taille restreinte, n'ont cependant pas intérêt à se livrer à une concurrence ou à une compétition dans le marché de l'emploi. Les groupes des DC adoptent plutôt une attitude d'inclusion (Olson, 1978 [1965], p. 59), à l'instar de l'Union des cadres supérieurs chômeurs qui a coordonné ses actions avec d'autres groupes (le Groupe Amal et le Groupe des cinq des cadres nationaux en chômage) pour observer ensemble un sit-in à Rabat. La nouvelle organisation élargie de la protestation a intégré par la suite le Groupe national des handicapés en chômage et les groupes des non-voyants afin de mener ensemble un sit-in devant le siège du Parlement.

Cependant, face aux groupes déjà constitués, disposant d'une légitimité liée à l'ancienneté, le dernier groupe créé s'expose, à chaque fois, à des difficultés à s'imposer et à intégrer l'équipe des interlocuteurs qui négocie avec les représentants du gouvernement. Il faut également noter que le groupe, bien que de dimension trop restreinte, reste autonome et peut agir seul, non sans une radicalisation parfois extrême. Ainsi, une vingtaine de non-voyants chômeurs ont-ils, par exemple, organisé trois semaines durant un sit-in dans la gare routière de Rabat avant de durcir leur mouvement en lançant une grève de la faim. Malgré le dialogue ouvert avec les instances officielles, notamment avec la secrétaire d’État chargée des handicapés, et après I08 jours en tout de sit-in, le groupe des non-voyants a finalement décidé d'organiser une manifestation dans les rues de Rabat en s’attachant par une chaîne de fer pour éviter leur dispersion par les forces de l'ordre. 


\section{LE CHOIX RATIONNEL: LE COÛT ET LES BÉNÉFICES}

$\mathrm{Si}$ au niveau de son organisation, un groupe restreint est certes facilement gérable et donc mobilisable, les chances de réussite d'une mobilisation sociale des DC n'en dépendent pas moins du nombre de protestataires. Or un certain nombre d'individus ne souhaitent pas participer à l'action collective dans un contexte politique autoritaire de peur d'être trop visibles dans l'espace public ou d'être fichés, réprimés ou arrêtés par la police et condamnés lourdement par la justice, ou tout simplement de peur d'être mal-considérés. Les jeunes DC tentent donc d'abord de réactiver leurs réseaux familiaux et amicaux pour pouvoir trouver un emploi convenable. La construction d'un groupe d'intérêt ou l'adhésion aux mouvements protestataires n'intervient qu'en second recours pour tenter d'accéder au marché de l'emploi ou plus exactement à la fonction publique.

Nous avons signalé que le groupe se crée par le biais d'une liste fermée où figurent les noms des participants à la protestation. Les nouveaux DC qui ne sont pas inscrits sur la liste, doivent former un nouveau groupe qui assurera, à son tour, sa propre organisation et action collective.

L’analyse de la réussite de la mobilisation du mouvement des jeunes DC peut se faire d'une manière pertinente grâce au paradigme du choix rationnel qui met l'accent sur la rationalité instrumentale de l'acteur et ses motivations utilitaires individuelles. À cet égard, «la croyance courante qui veut que des groupes de personnes ayant des intérêts communs tendent à les défendre semblent avoir peu ou pas de fondement» (Olson, ibid., p. 23).

En fait, l'organisation de l'action collective du mouvement étudié semble tirer les leçons de la théorie du choix rationnel. Le calcul de l'individu en termes de coûts/avantages semble devenir une source explicative de la participation à l'action collective. Le DC calcule doublement le coût de son éventuelle participation. Il sait que l'occupation de l'espace public sans autorisation administrative préalable l'expose au danger (intimidation, coups et blessures, arrestation, condamnation, torture, etc.) mais il mesure également la probabilité du succès de l'action collective; autrement dit, il calcule le bénéfice personnel qu’il va tirer de sa participation, à savoir l'obtention d'un emploi. 
L'organisation du groupe est faite de telle sorte que le DC ne peut en aucun cas profiter des résultats d'une action collective sans y participer. En effet, pour éviter «le passager clandestin», les mobilisateurs ont mis en œuvre des mécanismes d'incitation et de contrainte pour pousser l'individu «rationnel» à s'engager dans l'action collective. Leur capacité réflexive s'inscrit au niveau discursif (oral et écrit) et au niveau de l'organisation du groupe. Ils ont ainsi élaboré un statut détaillé qui fixe les règles de fonctionnement régissant le groupe des DC sous forme d'un ensemble de droits et d'obligations. Il s'agit d'un système de gratifications et de sanctions : les mobilisateurs distribuent des points en fonction de la nature de l'engagement et de la fréquence de la participation du jeune DC à l'action collective. Pour devenir un membre effectif du groupe, il faut, par exemple, participer à au moins cinq sit-in dans l'espace public. L'importance numérique des points accumulés permet aux membres du groupe d'être parmi les candidats prioritaires aux futurs postes d'emplois proposés par les autorités publiques, ce qui permet non seulement d'évincer le «passager clandestin» mais assure en outre une mobilisation quasi-quotidienne des DC dans les rues de Rabat.

Le statut élaboré par le Groupe des cadres supérieurs en chômage stipule dans son article ig que le membre du groupe peut bénéficier d'une autorisation pour s'absenter s'il est malade, à condition qu'il présente un certificat médical qui ne dépasse pas trois jours d’absence, sinon il doit présenter un dossier médical (des analyses, des radios, des certificats médicaux avec les cachets du médecin et du pharmacien validés par le ministère de la Santé4.) Un membre du groupe ne peut s'absenter que dans les cas suivants : après des coups ou blessures pendant les «actions militantes », après le décès d'un proche parent ou en cas de maladie.

Le statut fondateur du Groupe baptisé al-Ietissame (sit-in), stipule, quant à lui, dans son article 24 que l'exclusion de la liste du groupe concerne «tout adhérent qui :

- s'absente quatre fois sans justification;

- a déjà eu deux avertissements ;

4 Pour plus de détails voir en annexe des extraits du statut fondateur du Groupe des cadres supérieurs en chômage. 
- contourne le règlement intérieur du Groupe ;

- s'inscrit dans d'autres groupes ».

Cependant l’adhérent exclu peut réintégrer le Groupe en se réinscrivant. Il ne devient membre effectif qu'après sa participation à sept sit-in de suite. Concernant l'exclusion définitive, l’article 25 stipule que le Bureau prend la décision d'exclusion définitive dans les cas suivants :

- - après deux exclusions ;

- - après une absence sans justification pendant neuf sit-in de suite.

Cette organisation statutaire réserve la priorité au niveau d’une éventuelle embauche dans la fonction publique à ceux qui militent davantage dans les rues de Rabat. Comme nous l'avons signalé, ceci permet de contourner le problème de «passager clandestin » provenant essentiellement du caractère collectif des bénéfices. Il s’agit ainsi de mettre en pratique le principe du système du syndicalisme obligatoire (closed shop ${ }^{5}$ ) qui interdit l'accès à l'emploi aux non-syndiqués.

Le mouvement des DC inscrit donc son action collective dans une logique comptable. Le groupe distribue à ses membres des notes en fonction de la présence régulière dans les sit-in, de la nature des actions menées sur le terrain, de la gravité des coups et blessures reçus lors de l'intervention des forces de l'ordre, etc. En effet, le DC cherche à maximaliser les «bonnes notes» qui lui permettent d'être prioritaire au niveau de l'accès à l'emploi dans la fonction publique. Cette conception instrumentale de la rationalité ne doit pas cacher le fait que le DC a également besoin de tisser des liens sociaux avec des individus qui partagent les mêmes soucis, voire la même habitation. Des amitiés se créent à tel point qu'après leur embauche, certains DC ont pensé sérieusement à fonder une association des anciens diplômés chômeurs.

5 Le syndicalisme obligatoire est un accord ou un arrangement entre l'employeur et l'organisation syndicale qui contraint l'employé à être membre d'un syndicat pour être embauché. Le principe est qu'un salarié non-syndiqué ne peut bénéficier des résultats obtenus par l'action collective de ses collègues syndiqués. Critiqué par les libéraux comme attentatoire à la liberté contractuelle et celle de travailler, le closed shop a été interdit aux États-Unis en 1947 par la Loi Taft-Hartley. Margaret Thatcher l'a restreint progressivement au Royaume-Uni par des Employment Acts successifs en 1980, 1982, 1984, 1987 et 1988. L'Employment Act de 1990 a aboli strictement le closed shop, jugé attentatoire à la liberté de ne pas se syndiquer, garantie par la Charte sociale européenne. 
Mais le choix rationnel de l'individu n'est pas forcément raisonnable. Ainsi d'un jeune DC, docteur en histoire, responsable de la Communication de l'un des quatre principaux groupes. Deux mois après avoir été recruté dans un grand centre moderne privé de documentation à Casablanca, il déposa sa démission parce qu'il n’arrivait plus à assurer sa présence régulière dans les actions menées par son groupe à Rabat. Il risquerait donc d'être exclu de la liste du groupe. Grâce à sa participation permanente à l'action collective dans l'espace public, il est actuellement enseignant dans un lycée public à Casablanca.

Forts de la réussite des groupes antérieurs, les nouveaux DC sont convaincus qu'ils obtiendront tôt ou tard un emploi dans la fonction publique. Il leur suffit de persévérer dans la perturbation de l'ordre public. La règle devient la suivante: pour s'assurer un poste dans la fonction publique, il faut d'abord en passer par un processus de protestation dans les rues de Rabat. Cependant, d'autres contraintes interviennent, telles l'effet de la conjoncture politique, qui limite la portée du choix rationnel et de la volonté du DC.

Un autre exemple similaire nous vient d'une jeune femme préparant son doctorat en histoire qui venait nous demander conseil parce qu'elle narrivait pas à trancher entre deux préférences qui exigent une présence physique obligatoire : continuer à travailler dans le centre de documentation à Casablanca ou participer à la protestation dans les rues de Rabat. Elle doit répondre à la question suivante : quel est le meilleur moyen pour arriver à ses fins, c'est-à-dire l'obtention d'un emploi stable? Après une mûre réflexion, elle a également décidé de quitter son travail à Casablanca pour rejoindre les rangs des protestataires devant le siège du Parlement. Mais, malheureusement pour elle, sa décision s'est avérée préjudiciable pour sa carrière puisqu'elle se trouva dans l'impossibilité de réintégrer son emploi initial.

Sans aller jusqu'à prôner une explication de type holiste, nous pensons que le paradigme du choix rationnel n'est qu'une étape dans l'analyse de la participation à l'action collective. Certes, il permet d'expliquer d'une manière pertinente une partie du phénomène étudié, mais il n’a pas pour objectif de rendre compte de la réalité dans toute sa complexité. Lapproche de la mobilisation doit prendre en charge plus largement les effets des structures sociales, politiques, etc. 
À cet égard, la théorie de l’action collective (Oberschall, 2000, p. 25-35) permet de distinguer les différentes dimensions macro et micro susceptibles de construire la protestation sociale. Le niveau macro fait référence aux opportunités politiques locales, nationales, voire internationales favorables à l'organisation de la protestation sociale (liberté d'expression, accès aux mass-media, degré du mécontentement social, etc.) Le niveau micro met en exergue la capacité des acteurs à réussir la mobilisation sociale, la nature des biens collectifs à revendiquer, les objectifs de la protestation, les coûts de la participation (affrontement physique avec la police, arrestation, torture, intimidation, condamnation, etc.) et les bénéfices que le protestataire pourrait tirer de sa participation.

\section{LA FRUSTRATION, \\ LE PREMIER PAS VERS LE MÉCONTENTEMENT}

Contre une interprétation marxiste dominante qui cherche à expliquer la révolte par les crises économiques et la misère de la population, une hypothèse tend à émerger selon laquelle les gens se révoltent en situation de prospérité. À en croire l'analyse de Timothy Snyder et de Charles Tilly (1986) relative au cas de la France entre I830 et 1960, «la violence n’est ni plus intense, ni plus probable lorsque la rigueur des conditions de vie est plus grande» (voir Boudon, 1985, p. 85). Il ne faut donc pas sattendre à observer des relations causales mécaniques entre l'intensité du mécontentement social et celle de son expression collective violente (ibid., p. 79-90).

Comme l'écrivait Tocqueville :

Ce n'est pas toujours en allant de mal en pis que l'on tombe en révolution. Il arrive le plus souvent qu'un peuple qui avait supporté sans se plaindre, et comme s'il ne les sentait pas, les lois les plus accablantes, les rejette violemment dès que le poids s'en allège. Le régime qu'une révolution détruit vaut presque toujours mieux que celui qui l'avait immédiatement précédé, et l'expérience apprend que le moment le plus dangereux pour un mauvais gouvernement est d'ordinaire celui où il commence à se réformer (Tocqueville, 1986 [1856], p. 1058). 
La théorie de la frustration met en exergue le paradoxe entre la position sociale attendue et espérée du DC, après de longues études universitaires, et la réalité sociale amère qui le réduit au chômage, à la misère ou à l'exercice d'un métier qui ne valorise guère ses compétences acquises à l'université. Les attentes déçues provoquent un sentiment de frustration. Celui-ci est le premier pas vers l'expression du mécontentement.

L'espoir d'un éventuel changement politique nourrit donc la protestation sociale. Et, en effet, l'avènement du «gouvernement d'alternance » a contribué à l'essor des mouvements revendicatifs d'une manière générale. Les mouvements des DC n'ont pas attendu longtemps pour interpeller directement le nouveau gouvernement ${ }^{6}$. Onze jours seulement après sa nomination, l'Association nationale des DC a observé un sit-in devant le siège du Parlement le 25 mars 1998. Malgré la présence impressionnante des forces de l'ordre, le sit-in s'est déroulé sans aucun incident.

Pire encore, tandis qu'en 20II, le gouvernement promettait officiellement l'embauche de de près de $4000 \mathrm{DC}$ dans la fonction publique pour le budget de l'année 20I2, le nouveau gouvernement islamiste élu annulait finalement cette décision en imposant le recours préalable «aux concours ouverts à tous les citoyens » pour l'embauche dans la fonction publique, générant, là encore, un surcroît de frustration parmi les DC.

La notion de «al-hogra» qu’on peut traduire littéralement par «mépris», exprime un sentiment de frustration des DC qui ont entretenu l'espoir d'obtenir un emploi correspondant à leur statut intellectuel. De plus en plus utilisée par le discours protestataire, la notion de «al-hogra» exprime le sentiment de ne pas être perçu socialement à sa juste valeur, certains jeunes DC se sentant dévalorisés et inutiles. Les slogans revendicatifs s’appuient ainsi sur trois notions principales qui tendent à se confondre : la marginalisation, l'exclusion et la dignité (al-karama) qui est la version positive d'«al-hogra». Celle-ci peut traduire le concept de frus-

6 La formation gouvernementale était politiquement très hétérogène. En plus des ministères dits de souveraineté, d'autres partis politiques dits du centre participent également à ce nouveau gouvernement. Au total, sept partis politiques sont représentés dans ce gouvernement dit «d'alternance», mené par le parti de l'Union socialiste des forces populaires (USFP). 
tration qui est un concept central permettant d’analyser le mécontentement individuel des jeunes DC. La frustration est analysée par Gurr (2010) comme un état de tension, une satisfaction attendue et refusée, génératrice d'un potentiel de mécontentement et de violence. Le sentiment de frustration devient donc le premier pas vers la révolte tandis que la spirale inflationniste des attentes est susceptible de radicaliser le mécontentement social. L’intensité de la frustration peut ainsi augmenter le degré de la violence collective.

\section{VERS LA RADICALISATION DU MOUVEMENT}

Le recrutement massif des DC dans la fonction publique est perçu par le mouvement comme le fruit de leurs actions collectives récurrentes. Ainsi, non seulement les DC espèrent un éventuel recrutement à l'instar de leurs anciens collègues ${ }^{7}$, mais ils sont convaincus que leur stratégie de perturbation dans l'espace public aboutira fatalement à l'obtention d'un emploi. On peut donc comprendre que la déception est grande chez les DC quand ils se heurtent au refus des pouvoirs publics de satisfaire leurs revendications.

Depuis 1996, le mouvement des DC se radicalise: des sit-in et des grèves de la faim prolongées, un investissement violent des bâtiments officiels et des sièges des partis politiques au pouvoir, une perturbation de la circulation et des activités cérémonielles du gouvernement, des tentatives de suicide dans l'espace public en s'immolant par le feu, etc. Après un sit-in de neuf mois, accompagné d’une grève de la faim au siège du syndicat de l'Union marocaine du travail (UMT), une animatrice éducatrice s'est donnée la mort ${ }^{8}$, chez elle à Rabat, au mois de juin 2003. Les DC qui désespèrent et n’arrivent plus à assumer l'humiliation sociale se suicident ainsi loin des regards dans l'intimité de l'espace privé, exprimant par là plutôt un échec individuel d'intégration sociale qu’un acte de protestation sociale.

7 Le ministre délégué chargé de la modernisation des secteurs publics a déclaré que l'État a créé, en trois ans, 4300 postes dans la fonction publique en faveur des diplômés universitaires chômeurs du supérieur, soit I 250 postes en 2009, I 513 en 2010 et I 537 en 20 II.

8 Le premier suicide d'un diplômé universitaire (en chômage) de la Faculté des Sciences de l'éducation date de 1996. Un autre jeune de 29 ans, licencié en Biologie, en chômage depuis quatre ans et membre actif au sein de l'Association des diplômés en chômage à Dchira (Agadir), se donna également la mort en 1998. 
Moins médiatisé, moins spectaculaire, cet acte suicidaire est par conséquent sans effet ni sur la politique publique en matière d'emploi ni sur l'opinion publique.

Cependant, dès le début des années 2000, le mouvement des DC passe à une autre étape pour exercer sa pression politique sur le gouvernement: le suicide collectif dans l'espace public. Sachant que l'État est davantage préoccupé par l’amélioration de son image de marque en manifestant sa volonté politique à veiller au respect des droits de l'homme, les jeunes DC ont usé et usent encore de plusieurs moyens de pression.

La stratégie de perturbation récurrente de l'ordre public est mise en ouvre en sattaquant avant tout aux bâtiments des ministères et aux sièges des partis politiques représentés au gouvernement. Environ i80 DC ont ainsi investi le siège de l'annexe du ministère de l'Éducation nationale en 20I2. Après douze jours de sit-in, trois DC se sont immolés par le feu, devant ce même siège, dont l'un décèdera finalement à l'hôpital. Dès lors, les forces de l'ordre n'hésitent plus à disperser violemment les DC, les arrêter et les déférer en justice. Après plusieurs actions de protestation sous forme de sit-in, un groupe en chômage de 34 jeunes lauréats du DESA de l'année 2000-200I, baptisé groupe al-Wafaa, menaça de s'immoler par le feu dans la rue'.

La radicalisation de l'action collective est également engagée par d’autres mouvements protestataires. Les enseignants se trouvant dans l'impossibilité de joindre leurs conjoints ont lancé une grève de la faim le in septembre 2005, avec une série de sit-in devant le siège du ministère de l'Éducation nationale et au siège du syndicat d'al-Jamiaa al-horra littaalim à Rabat. Les forces de l'ordre sont intervenues d'une manière violente pour disperser les protestataires. Sur les 40 enseignants, la presse signale que 23 d'entre eux ont été hospitalisés à cause de la détérioration de leur état de santé. Un autre mouvement social similaire est engagé en 20ı0. Revendiquant le droit du groupement familial, les enseignantes ont poursuivi leur grève de la faim qui a duré 57 jours.

9 Vers la fin de l'année 2004, un militant au chômage depuis quatre ans, muni d'une corde, a tenté de mettre fin à sa vie lors d'un sit-in devant le siège du Parlement et devant une présence massive des forces de l'ordre. 


\section{LA FRUSTRATION, UN PRODUIT DE LA POLITIQUE PUBLIQUE}

Pendant des décennies, la situation de pauvreté sociale et d'exclusion spatiale était vécue comme «naturelle» par la population. Certes, des revendications sociales existaient, mais elles étaient sporadiques, spontanées, ponctuelles et isolées. Conformément à la théorie de la frustration relative, «quand les gens sont dans un cas d'extrême désespoir et de misère, ils sont en général peu enclins à la révolte, parce qu'ils sont sans espoirs [...] Mais quand leurs situations s'améliorent et ont le sentiment d’un éventuel changement, ils se révoltent positivement contre l'oppression et l'injustice» (Gurr, 2oı, p. I I4).

La frustration relative provoquée par une perception collective d'une éventuelle amélioration des conditions de vie est donc au fondement du mécontentement individuel. Le processus d'ouverture du système politique accompagnant la mise en œuvre (et la médiatisation) d'une politique publique de grande envergure contre la pauvreté à partir de 2004 (le programme d'Initiative nationale pour le développement humain, la politique massive du logement social pour le recasement des ménages des bidonvilles, l'introduction de l'électrification en milieu rural, l'élaboration de nouveaux régimes de couverture médicale pour les démunis, etc.) est, de ce point de vue, un élément déterminant dans l'augmentation de l'intensité de la protestation.

En effet, en 2005, l'action collective des différents mouvements protestataires dans l'espace public (sit-in, manifestation, marche, etc.) se sont traduites en 700 sit-in, soit une moyenne de deux sit-in par jour. En parallèle à l'engagement d'une politique publique multidimensionnelle, les protestations ont donc augmenté très sensiblement. Elles ont passé de 5000 actions en 2008 à 6438 en 2009 pour atteindre 8600 actions en 2010 (données statistiques fournies par le Ministère de l'intérieur, rabat, 20I I). La récurrence des actions du Mouvement du 20 février, en 20ı I, dans le cadre du «Printemps arabe», à l'origine d'une augmentation vertigineuse du nombre de protestations. Sous le gouvernement mené par le PJD (Parti de la justice et de développement), le nombre de protestation en 2012 a été multiplié par 26 par rapport à l'année 2005. L'ensemble des participants à ces protestations est estimé à 32 I 000 personnes. Le chiffre de la 
protestation collective est en augmentation continue. Il atteint presque i8 600 protestations en 2013, soit une moyenne de 52 protestations par jour.

En cherchant à lutter contre le chômage des DC, le désenclavement rural et la pauvreté urbaine, la politique publique a également pour conséquence de nourrir l'espoir et de multiplier les attentes sociales. L'intervention de l'État dans des espaces enclavés et marginalisés est ressentie comme discriminatoire par les habitants des autres quartiers ou régions souffrant des mêmes difficultés. Elle provoque finalement un sentiment d'injustice et donc une frustration relative.

\section{UN RYTHME DE PROTESTATION EN BAISSE}

Laction collective au cours de l'année 20I4 connaît un tournant dans son rythme d'accroissement, le nombre de protestations accusant une baisse de plus de $40 \%$. Il est de l'ordre de 8500 protestations (enregistrées jusqu'au 30 septembre, données officielles du ministère de l'Intérieur), soit une moyenne de 3 I actions par jour en 2014 contre 52 en 20I3. Plusieurs facteurs peuvent expliquer cette baisse très sensible.

D’abord, de nouvelles opportunités politiques émergent qui vont structurer le champ protestataire. En effet, après une période de tolérance à l'égard des mouvements revendicatifs, liée au «Printemps arabe», en 20I I, l’attitude de l'État à l'égard des DC allait changer. Le nouveau gouvernement ${ }^{10}$, légaliste, issu des élections de 20I2, cherche à démontrer sa force répressive et sa capacité à maintenir et à assurer le retour rapide de l'ordre face à la «stratégie de perturbation» (Tbarzite) de l'ordre public menée par les DC.

S’appuyant sur sa légitimité électorale, le nouveau gouvernement revendique l'application de la loi quitte à adopter des mesures perçues socialement comme impopulaires ${ }^{11}$. On peut citer la position ferme du nouveau gouvernement à l'encontre des groupes des DC qui exigent l'application du « PV du 20 juillet 2o I » signé avec le Premier ministre de l’ancien gouvernement, pour

10 Le gouvernement est mené par le parti islamiste, le Parti de la justice et de développement (PJD).

II Nous pouvons aussi signaler dans ce sens, le prélèvement du gouvernement sur les salaires des fonctionnaires grévistes, malgré les multiples protestations des syndicats. 
le budget de l'année 20I2. Les DC reprochent au nouveau gouvernement, mené par le PJD, de ne pas respecter les principes fondamentaux des engagements de l'État. Selon le principe défendu par le gouvernement contre les DC, l'accès à la fonction publique ne doit en aucun cas passer par la protestation, mais par la voie légale, laquelle impose à tous les citoyens de passer un concours permettant la sélection des individus compétents et non des militants.

La majorité des protestations collectives, estimées officiellement à 80\%, ne disposant pas d'autorisations administratives préalables leur permettant d'occuper l'espace public, le nouveau gouvernement peut, quant à lui, justifier son action répressive au motif qu'il est élu pour appliquer la loi. Cependant l'action répressive touche également certaines associations, issues des anciens mouvements politiques d'extrême gauche, qui travaillent dans la légalité. De fait, l'État est en passe de durcir sa position à l'égard de certaines associations des droits de l'homme. Depuis juillet 20I4, 48 activités de l'Association marocaine des droits humains (AMDH) ont été ainsi interdites en trois mois et demi, dont sept sit-in dans l'espace public.

Le second facteur explicatif de la baisse du rythme de la protestation est lié à la diminution de l'ampleur de la politique publique massive engagée depuis 2005. En effet, le recasement de milliers de ménages bidonvillois a été accompli, et les protestations que la politique du logement social avait provoquées, ont été en grande partie réglées. L’espoir et les attentes qu’a suscités la politique publique massive s'estompent ainsi progressivement avec le temps. Quoique l'agrégation des frustrations individuelles ne débouche pas forcément sur une action collective, le mécontentement individuel n'en est pas moins toujours présent. Mais sans opportunités politiques favorables à la construction de la protestation, le sentiment d'injustice a peu de chance de se transformer en action collective organisée.

La théorie de la mobilisation des ressources offre de surcroît un cadre théorique permettant d'envisager une autre approche des mouvements protestataires. Pour que le sentiment de frustration se transforme en action collective, il a également besoin d'acteurs capables de mobiliser des individus mécontents. Des acteurs disposant de compétences intellectuelles, d'expérience et de savoir-faire militants, qui savent négocier, parler en public, gérer les conflits internes, et qui peuvent accéder aux mass-média et mobiliser les organisations politiques et 
syndicales. Il ne s'agit plus comme dans le modèle dit de «collective behaviour » de se demander pourquoi des groupes se révoltent, mais comment se déclenche, se développe, réussit ou échoue une mobilisation collective (Offerlé, 1994, p. 52).

En effet, la seule théorie de la frustration ne permet pas d'expliquer le processus du passage du mécontentement individuel (sentiment d'injustice) à l'action collective. Elle ne prend pas en charge la nature des contraintes qu'exerce le système politique sur l'action collective. Elle ne cherche pas non plus à expliquer l'évolution progressive des formes de protestation sociale ni le changement de la nature de l'action collective. La prise en compte de la structure autoritaire du pouvoir et des opportunités politiques (nationales, régionales ou internationales) est incontournable dans l'analyse de la protestation sociale.

Si l'ouverture du système politique favorise la construction de la protestation sociale, cette dernière n'est pas pour autant le produit pur et simple de ce contexte politique. L'émergence de la société civile - notamment les mouvements des droits de l'homme, les mouvements de femmes, le mouvement des diplômés chômeurs, etc. - , s'est réalisée dans un contexte politique autoritaire. Les mouvements sociaux exercent également des pressions sur l'État par le recours à la grève générale ou sectorielle qui aboutissait à l'émeute.

\section{CONCLUSION}

Pour approcher la dynamique de la protestation sociale au Maroc, nous nous sommes référé à plusieurs théories et paradigmes sociologiques. Ces derniers nous ont servi de base pour mieux mener l'analyse de la protestation sociale. La théorie de la mobilisation des ressources n'est nullement incompatible avec la théorie de la frustration relative, ni avec le paradigme du choix rationnel ou les mouvements sociaux émotionnels. On ne peut même pas faire l'économie de certaines approches psychologisantes (Le Bon, I983 [I895] ou Gurr, 20ı, par exemple). Un seul paradigme ne saurait rendre intelligible à lui seul l'évolution et l'hétérogénéité des formes sociales de protestation dans un contexte politique en changement. 
Parce que la mise en œuvre d'une action collective ne peut se réduire au simple sentiment de frustration ou aux mécanismes d'incitation et de contrainte, il faut également prendre en considération la capacité des acteurs à mobiliser les DC. L'accent doit être enfin mis sur la volonté politique de l'Etat à réprimer, à tolérer, à contrôler ou à contenir un mouvement en ébullition.

En l'absence d'une tradition de protestation sociale urbaine pacifique, les émeutes se sont multipliées dans plusieurs villes marocaines tout au long des années 1980. Le passage de l'émeute à l’action manifestante correspond également au passage du temps de la violence militaire (aveugle) à celui de la violence policière et du temps du marchandage à celui de la négociation et du dialogue.

Étant à l'initiative de nouvelles formes pacifiques de protestation sociale dans l'espace public, les DC ont réussi à créer une tradition, relativement récente, de la manifestation dans l'espace public. En parallèle, d'autres nouveaux mouvements revendicatifs liés à la promotion de valeurs (défense des droits de l'homme, des femmes, de la culture amazighes, etc.) se sont renforcés. Mais, il a fallu attendre l'année 2000 pour pouvoir assister à une manifestation dont les enjeux politiques sont liés directement à la situation interne du pays. En effet, en accord avec les autorités locales, deux manifestations ont été organisées en même temps, l'une par le courant islamiste, à Casablanca, l'autre, par les différentes organisations dites progressistes et modernistes, à Rabat.

Depuis ces deux gigantesques manifestations quá connues le Maroc à l'occasion de la journée mondiale des droits de la femme, le I2 mars 2000, les protestations sociales prennent de plus en plus des dimensions politiques en clivant le pays en deux blocs: un bloc dominé par les islamiste et un autre par les modernistes.

Ainsi, les manifestations massives pendant le «Printemps arabe», en 20II, se sont inscrites dans cette tradition de protestation pacifique. Laction collective hebdomadaire (sit-in et manifestation) du Mouvement du 20 février au Maroc, tout au long de l'année 200I, est le produit d'un long processus de protestation sociale. Les acteurs mobilisateurs ont réussi à grouper plusieurs tendances politiques hétérogènes afin de dénoncer l'ordre socio-politique établi. Malgré l'intervention parfois répressive des forces de l'ordre, ces manifestations ne se sont jamais transformées en émeute. 
L'évolution de l'action collective dans l'espace public au Maroc traduit donc le passage du temps de la protestation sociale émotionnelle à celui de la revendication d'intérêts catégoriels, puis de l'émergence de nouveaux mouvements sociaux liés à la promotion de valeurs (défense des droits de l'homme, des femmes, de la culture amazighes, etc.) à la contestation plus générale du système politique dans l'espace public.

NdA. Je tiens à remercier Hassan Rachik et Mohamed Cherkaoui pour leurs remarques et critiques qui ont été d'un grand intérêt pour la rédaction du présent article.

\section{BIBLIOGRAPHIE}

BOUDON R., 1985, La Place du désordre, Paris, PUF.

CHERKAOUI M., 2003, «L'État et la Révolution: logique du pouvoir monopoliste et mécanismes sociaux dans L'Ancien Régime de Tocqueville», The Tocqueville review / La Revue Tocqueville, 24-I, p. 163-187.

DAVIES J.C. (ed.), 1997, When Men Revolt and Why, New Brunswick, London, Transaction Publishers.

GURR T.R., 2010, Why Men Rebel, Boulder, London, Paradigm Publishers.

LE BON G., 1983 (1895), Psychologie des foules, PUF.

OBERSCHALL A., 2000, «Social Movement and the Transition to Democracy», Democratization, 7-3, p. 25-35.

OFFERLÉ M., 1994, Sociologie des groupes d'intérêt, Paris, Montchrestien.

OLSON M., 1978 (1965), Logique de l'action collective, Paris, PUF.

RACHIK A., 2014, Les Mouvements de protestation au Maroc, de l'émeute à la manifestation, Publication du Forum des alternatives Maroc (Rabat).

TILLY Ch., 1986, La France conteste de 1600 à nos jours, Paris, Fayard.

TOCQUEVILLE A. (de), 1986 (I856), L’Ancien Régime et la Révolution, in Id., De la Démocratien en Amérique, Souvenirs, L’Ancien Régime et la Révolution, Paris, Robert Laffont. 


\section{ANNEXE \\ QUELQUES EXTRAITS DU STATUT FONDATEUR DU GROUPE AL-IETISSAME (SIT-IN) DES CADRES SUPÉRIEURS EN CHÔMAGE}

Article 2: Objectif

Le Groupe al-letissame vise à parvenir à une intégration directe et immédiate des cadres supérieurs dans les différentes filières de la fonction publique.

\section{Chapitre II: L’adhésion}

Article 4: Les conditions d'adhésion

Pour participer au Groupe des cadres supérieurs en chômage, les conditions suivantes doivent être remplies:

- Être titulaire d'un Doctorat, Master, Diplôme d'études supérieures spéciali sées ou approfondie, en génie, etc. ou équivalent. Les diplômés à l'étranger doivent fournir l'équivalent officiel du diplôme;

- être enregistrés auprès du ministère de l'emploi et de la formation professionnelle comme cadre en chômage;

- payer la cotisation de 120 dirhams.

Article 5.

Pour devenir un membre effectif du groupe, il faut assister à cinq sit-in depuis la date de l'adhésion

\section{Chapitre IV: Système de contrôle}

- Chaque membre du groupe doit participer aux formes régulières de lutte, soit un taux de $75 \%$ de présence et $25 \%$ d'absence, l'équivalent de 3 jours par mois sans aucune justification. Mais pour les principales mobilisations, la présence est obligatoire à 100\%, en prenant en considération les cas spéciaux après autorisation du bureau;

- chaque mois, la liste est mise à jour ou à chaque fois que le besoin s'en fait sentir.

Les membres du groupe peuvent bénéfice d'une autorisation d'absence dans les cas suivants:

I. Autorisation est fournie par le Bureau en cas de maladie qui ne dépasse pas avec présentation d'un certificat médical qui ne dépasse pas 3 jours, mais quand le temps de la maladie dépasse trois jours, il faut fournir un dossier médical composé des documents suivants: certificat d'examen, les radios et les analyses. Le certificat médical avec le cachet du médecin et du pharmacien, les prospectus des médicaments (légalisés par le ministère de la Santé Publique).

Ce dossier médical doit être présenté au comité de suivi médical du Groupe dans un délai qui ne dépasse pas les sept jours après la date déclarée de la maladie. 
2. Autorisation pour chaque membre du Groupe blessé pendant l'organisation des actions protestataires. Le comité de contrôle et d'aide lui rend visite en considérant son état de santé pour permettre l'allongement de la durée de la période autorisée.

3. Autorisation de s'absenter pendant un mois après le décès de l'un des parents ou des sœurs et frères.

4. Autorisation de s'absenter pendant une semaine après le décès de l'un de ses proches (à partir du jour du décès): oncle, tante, etc.

5. Autorisation de s'absenter en cas d'hospitalisation urgente, à condition qu'elle ne dépasse pas les 15 jours. Le bénéficiaire doit soumettre une demande écrite au comité du Groupe la Commission, accompagnée d'un certificat médical.

6. Le droit pour chaque adhérent de profiter d'un nombre de jours limité, déterminé par le comité du Groupe afin de passer des concours d'emploi ou pour s'inscrire en doctorat, après avoir soumis une demande au comité, accompagné de pièces justificatives.

7. Pour les cas différents de ceux mentionnés ci-dessus, l'adhérent doit fournir au comité de contrôle une demande justifiant son absence.

Article 24: La première exclusion

La première exclusion du Groupe concernetout adhérent qui:

- S'absente des sit-in quatre fois sans justification;

- a déjà deux avertissements;

- contourne le règlement intérieur du Groupe;

- adhère à d'autres groupes du mouvement des diplômés chômeurs.

L'adhérent exclu peut réintégrer le Groupe en se réinscrivant, il ne devient membre effectif qu'après sa participation à sept sit-in de suite.

Article 25: L'exclusion défınitive.

Le bureau prend la décision d'exclusion définitive dans les cas suivants:

- Après deux exclusions;

- absence non-justifiée pendant neuf (9) sit-in de suite. 